\title{
Statistical Signal Processing by Using the Higher-Order Correlation between Sound and Vibration and Its Application to Fault Detection of Rotational Machine
}

\author{
Hisako Masuike ${ }^{1}$ and Akira Ikuta ${ }^{2}$ \\ ${ }^{1}$ Department of Circulation Information, Hiroshima National College of Maritime Technology, Toyota-gun, \\ Hiroshima 725-0231, Japan \\ ${ }^{2}$ Department of Management Information Systems, Prefectural University of Hiroshima, Hiroshima 734-8558, Japan
}

Correspondence should be addressed to Akira Ikuta, ikuta@pu-hiroshima.ac.jp

Received 27 June 2008; Revised 16 September 2008; Accepted 10 November 2008

Recommended by Fidel E. Hernandez Montero

In this study, a stochastic diagnosis method based on the changing information of not only a linear correlation but also a higher-order nonlinear correlation is proposed in a form suitable for online signal processing in time domain by using a personal computer, especially in order to find minutely the mutual relationship between sound and vibration emitted from rotational machines. More specifically, a conditional probability hierarchically reflecting various types of correlation information is theoretically derived by introducing an expression on the multidimensional probability distribution in orthogonal expansion series form. The effectiveness of the proposed theory is experimentally confirmed by applying it to the observed data emitted from a rotational machine driven by an electric motor.

Copyright ( ) 2008 H. Masuike and A. Ikuta. This is an open access article distributed under the Creative Commons Attribution License, which permits unrestricted use, distribution, and reproduction in any medium, provided the original work is properly cited.

\section{INTRODUCTION}

Some studies on the diagnoses formany machines, for example, the estimation and prediction of presenceof crack in rotational machines without stopping the machines, have become important recently because of increased use of various complex industrial systems [1-9]. Most of these investigations have been carried out especially in a frequency domain by the use of signal information on sound or vibration wave emitted from the machines. These standard methods in a frequency domain are useful for the purpose of analyzing the failure of individual machine based on the mechanism from a bottom-up viewpoint. However, these methods require a lot of procedures and times on signal processing for measured sound or vibration data.

In the actual machine systems, simultaneous time series data on sound and vibration signals can be easily observed by the use of multichannel measurement instruments. The correlation statistics between the sound and vibration emitted from an identical machine contain important information on the driving condition of the machine. For the diagnosis of the fault without stopping the machine, it is necessary to consider various kinds of latent correlation information of not only the lower order but also the higher orders in order to investigate and evaluate precisely the mutual relationship between sound and vibration emitted from the machine.

The standard diagnoses in frequency domain seem to be inadequate for evaluating total effects on the compound of the mutual relationship between sound and vibration waves in complicated circumstances, such as the actual driving condition. In order to evaluate universally the mutual correlation characteristics and detect the failure of machines by the use of a simple procedure in the actual complex working environment, it is necessary to introduce some signal processing methods, especially in a time domain.

From the above viewpoints, in this theory, a diagnosis method based on a conditional probability reflecting the linear and nonlinear correlation information with lower and higher orders is proposed in order to grasp minutely and universally the mutual relationships between sound and vibration emitted from machines. More specifically, a conditional probability expression for multivariables is 


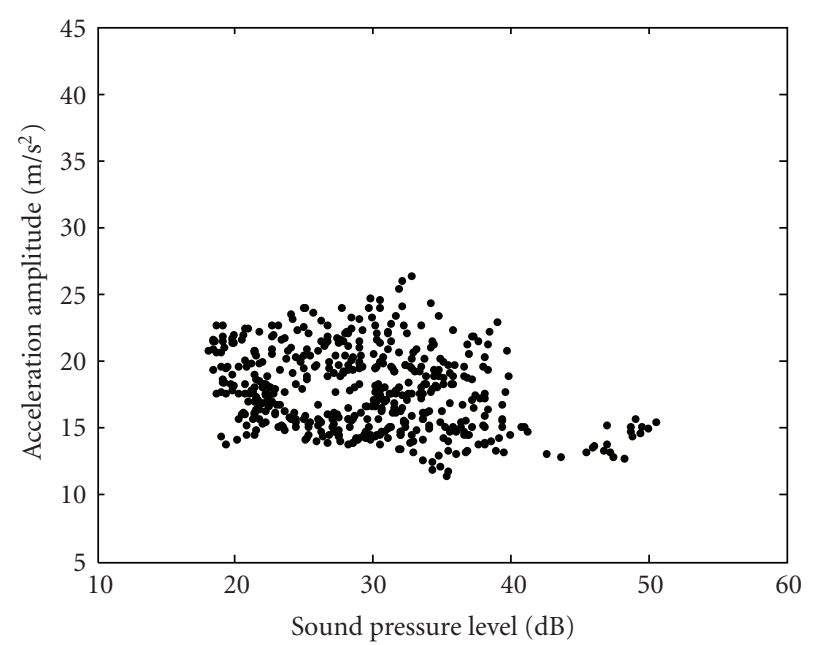

FIgURE 1: Scatter diagram between the observed data of sound and vibration in normal situation without the fault.

theoretically derived by introducing a multidimensional joint probability function in a series-type expression. First, in Section 2, based on the observation data of sound and vibration emitted from machines, an expansion series expression of the conditional probability distribution to evaluate the correlation relationship between the sound and vibration signals is theoretically derived. Next, in Section 3, by the use of the expansion expression of the conditional probability distribution derived in Section 2, a prediction method of the probability for a failure of machines is theoretically derived on the basis of the observation data in a sequential time series form of sound and vibration. The fundamental principle of the proposed diagnosis method is based on the detection of the changing information on various kinds of correlation characteristics between sound and vibration as much as possible. Finally, in Section 4, by applying the proposed methodology to the measurement data from a rotational machine driven by an electric motor, the effectiveness of theory is confirmed experimentally.

\section{THEORY}

\subsection{Conditional probability expression of fault occurrence by considering correlation relationship between sound and vibration}

Since there is a certain correlation relationship between sound and vibration emitted from an identical machine according to the driving condition of the machine, the diagnoses of the machine are possible by detecting the changing information of the correlation characteristics. First, let us introduce a random variable $y$ with two exclusive values of 0 and 1 corresponding to normal situation without fault of machines and a failure situation with the fault. In order to evaluate quantitatively the complicated relationship between sound and vibration emitted from a machine, let us introduce an expansion expression of conditional probability considering not only the linear correlation but also the

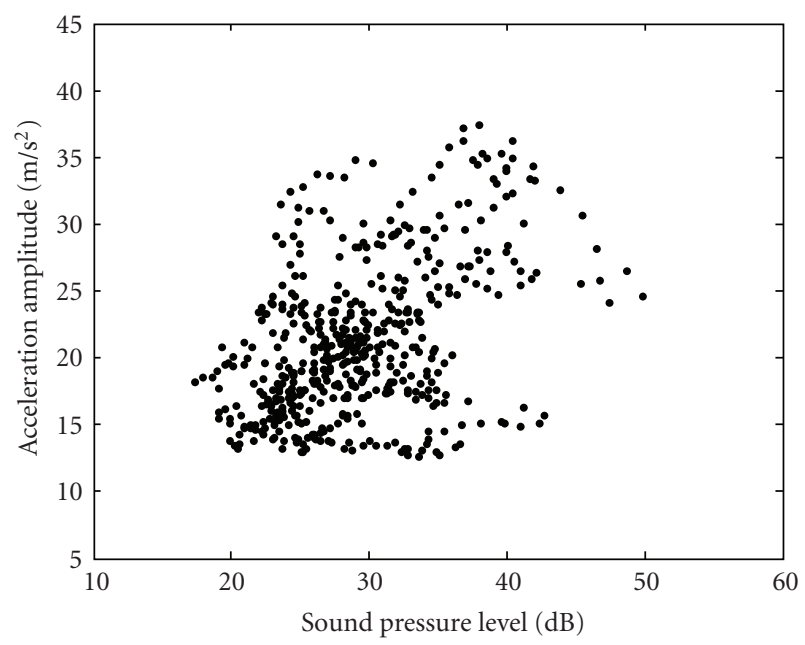

FIGURE 2: Scatter diagram between the observed data of sound and vibration in failure situation with the fault.

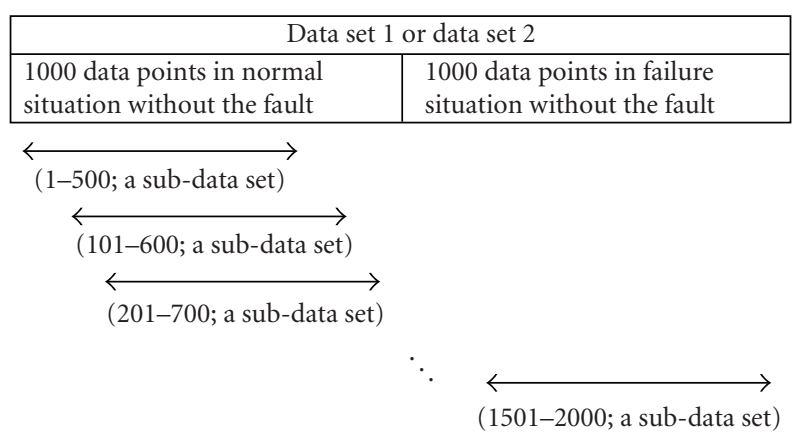

Figure 3: Relationship between Datasets and subdatasets.

nonlinear correlation information among these variables. In order to derive a mathematical expression in a general form, two kinds of variables (i.e., sound and vibration) are expressed as $x_{1}$ and $x_{2}$. In the case when paying attention to the variables $x_{1}, x_{2}$, and $y$, all the information on mutual correlations among $x_{1}, x_{2}$, and $y$ is included in the conditional probability distribution $P\left(y \mid x_{1}, x_{2}\right)$.

First, the joint probability distribution $P\left(x_{1}, x_{2}, y\right)$ is expanded into an orthonormal polynomial series [10] on the basis of the fundamental probability distributions $P_{0}\left(x_{1}\right)$, $P_{0}\left(x_{2}\right)$, and $P_{0}(y)$, which can be artificially chosen as the probability distributions describing approximately the dominant parts of the actual fluctuation pattern as follows:

$$
\begin{gathered}
P\left(x_{1}, x_{2}, y\right)=P_{0}\left(x_{1}\right) P_{0}\left(x_{2}\right) P_{0}(y) \sum_{m_{1}=0}^{\infty} \sum_{m_{2}=0}^{\infty} \sum_{n=0}^{\infty} A_{m_{1} m_{2} n} \\
\times \varphi_{m_{1}}^{(1)}\left(x_{1}\right) \varphi_{m_{2}}^{(2)}\left(x_{2}\right) \varphi_{n}^{(3)}(y), \\
A_{m_{1} m_{2} n}=\left\langle\varphi_{m_{1}}^{(1)}\left(x_{1}\right) \varphi_{m_{2}}^{(2)}\left(x_{2}\right) \varphi_{n}^{(3)}(y)\right\rangle,
\end{gathered}
$$

where $\langle\cdot\rangle$ denotes the averaging operation with respect to therandom variables. Here, $\varphi_{m_{1}}^{(1)}\left(x_{1}\right), \varphi_{m_{2}}^{(2)}\left(x_{2}\right)$, and $\varphi_{n}^{(3)}(y)$ are orthonormal polynomials with three weighting functions 
$P_{0}\left(x_{1}\right), P_{0}\left(x_{2}\right)$, and $P_{0}(y)$, and must satisfy, respectively, the following orthonormal relationships:

$$
\begin{gathered}
\int \varphi_{m_{1}}^{(1)}\left(x_{1}\right) \varphi_{m_{1}^{\prime}}^{(1)}\left(x_{1}\right) P_{0}\left(x_{1}\right) d x_{1}=\delta_{m_{1} m_{1}^{\prime}}, \\
\int \varphi_{m_{2}}^{(2)}\left(x_{2}\right) \varphi_{m_{2}^{\prime}}^{(2)}\left(x_{2}\right) P_{0}\left(x_{2}\right) d x_{2}=\delta_{m_{2} m_{2}^{\prime}} \\
\sum_{y=0}^{1} \varphi_{n}^{(3)}(y) \varphi_{n^{\prime}}^{(3)}(y) P_{0}(y)=\delta_{n n^{\prime}} .
\end{gathered}
$$

Thus, the information on the various types of linear and nonlinear correlations among $x_{1}, x_{2}$, and $y$ is reflected hierarchically in each expansion coefficient $A_{m_{1} m_{2} n}$.

Next, from (1), the following expression can be obtained:

$$
\begin{aligned}
P\left(x_{1}, x_{2}\right) & =\sum_{y=0}^{1} P\left(x_{1}, x_{2}, y\right) \\
& =P_{0}\left(x_{1}\right) P_{0}\left(x_{2}\right) \sum_{m_{1}=0}^{\infty} \sum_{m_{2}=0}^{\infty} A_{m_{1} m_{2} 0} \varphi_{m_{1}}^{(1)}\left(x_{1}\right) \varphi_{m_{2}}^{(2)}\left(x_{2}\right) .
\end{aligned}
$$

Thus, by using (1) and (4), the conditional probability distribution function containing all the information on the regression relationship can be derived under theemployment of the well-known Bayes' theorem [11] as follows:

$$
\begin{aligned}
& P\left(y \mid x_{1}, x_{2}\right) \\
& =\frac{P\left(x_{1}, x_{2}, y\right)}{P\left(x_{1}, x_{2}\right)} \\
& =\frac{P_{0}(y) \sum_{m_{1}=0}^{\infty} \sum_{m_{2}=0}^{\infty} \sum_{n=0}^{\infty} A_{m_{1} m_{2} n} \varphi_{m_{1}}^{(1)}\left(x_{1}\right) \varphi_{m_{2}}^{(2)}\left(x_{2}\right) \varphi_{n}^{(3)}(y)}{\sum_{m_{1}=0}^{\infty} \sum_{m_{2}=0}^{\infty} A_{m_{1} m_{2} 0} \varphi_{m_{1}}^{(1)}\left(x_{1}\right) \varphi_{m_{2}}^{(2)}\left(x_{2}\right)} .
\end{aligned}
$$

As the fundamental probability density functions $P_{0}\left(x_{1}\right)$ and $P_{0}\left(x_{2}\right)$ in the first terms of the expansion series expression of (1), the well-known Gaussiandistribution is adopted because this probability density function is the most standard one:

$$
\begin{aligned}
& P_{0}\left(x_{1}\right)=\frac{1}{\sqrt{2 \pi \sigma_{x_{1}}^{2}}} \exp \left\{-\frac{\left(x_{1}-\mu_{x_{1}}\right)^{2}}{\sigma_{x_{1}}^{2}}\right\}, \\
& P_{0}\left(x_{2}\right)=\frac{1}{\sqrt{2 \pi \sigma_{x_{2}}^{2}}} \exp \left\{-\frac{\left(x_{2}-\mu_{x_{2}}\right)^{2}}{\sigma_{x_{2}}^{2}}\right\}
\end{aligned}
$$

with

$$
\begin{array}{ll}
\mu_{x_{1}}=\left\langle x_{1}\right\rangle, & \sigma_{x_{1}}^{2}=\left\langle\left(x_{1}-\mu_{x_{1}}\right)^{2}\right\rangle, \\
\mu_{x_{2}}=\left\langle x_{2}\right\rangle, & \sigma_{x_{2}}^{2}=\left\langle\left(x_{2}-\mu_{x_{2}}\right)^{2}\right\rangle .
\end{array}
$$

Furthermore, as the fundamental probability function $P_{0}(y)$ on the level-quantized random variable $y$, the binomial distribution can be chosen [12]

$$
P_{0}(y)=\frac{((N-M) / h) !}{((y-M) / h) !((N-y) / h) !} p^{(y-M) / h}(1-p)^{(N-y) / h}
$$

with

$$
\begin{aligned}
& N=1 ; \text { the maximum value of } y, \\
& M=0 ; \text { the minimum value of } y, \\
& h=1 ; \text { the level difference interval of } y .
\end{aligned}
$$

Thus, from (3), the orthonormal functions can be determined as

$$
\begin{aligned}
& \varphi_{m_{1}}^{(1)}\left(x_{1}\right)= \frac{1}{\sqrt{m_{1} !}} H_{m_{1}}\left(\frac{x_{1}-\mu_{x_{1}}}{\sigma_{x_{1}}}\right), \\
& \varphi_{m_{2}}^{(2)}\left(x_{2}\right)= \frac{1}{\sqrt{m_{2} !}} H_{m_{2}}\left(\frac{x_{2}-\mu_{x_{2}}}{\sigma_{x_{2}}}\right), \\
& \varphi_{n}^{(3)}(y)= \frac{1}{\sqrt{((N-M) / h)^{(n)} n !}}\left(\frac{1-p}{p}\right)^{n / 2} \frac{1}{h^{n}} \\
& \times \sum_{j=0}^{n}\left(\begin{array}{c}
n \\
j
\end{array}\right)(-1)^{n-j}\left(\frac{p}{1-p}\right)^{n-j} \\
&(N-y)^{(n-j)}(y-M)^{(j)}
\end{aligned}
$$

with

$$
x^{(n)}=x(x-h)(x-2 h) \cdots(x-(n-1) h), \quad x^{(0)}=1,
$$

where $H_{m}(\bullet)$ is the Hermite polynomial with $n$th order [13]. By substituting (8)-(10) into (2) and (5), the conditional probability distribution function $P\left(y \mid x_{1}, x_{2}\right)$ can be obtained in the concrete form.

It may be natural to impose a condition $A_{001}=0$ upon the expansion coefficient in (2) in order to get an appropriate explanation for the dominant part of the probability distribution only by the first term. Thus, the unknown parameter $p$ can be explicitly determined by the use of mean value of the variable $y$ as

$$
p=\frac{\mu-M}{N-M}, \quad \mu=\langle y\rangle
$$

Furthermore, four parameters $\mu_{x_{1}}, \mu_{x_{2}}, \sigma_{x_{1}}^{2}$, and $\sigma_{x_{2}}^{2}$ in (6) can be determined as (7), by considering the following conditions on expansion coefficients

$$
A_{100}=A_{010}=A_{200}=A_{020}=0 .
$$

In the probability distribution expression of infinite series type, by adopting the probability distribution function describing the dominant parts of the phenomena as the fundamental probability distribution, it is enough to consider only a few expansion terms in order to express the whole fluctuation form. In order to roughly express the whole shape of the probability distribution function, it is fundamentally important to first catch the statistical information on two lower-order moments like mean and variance. 


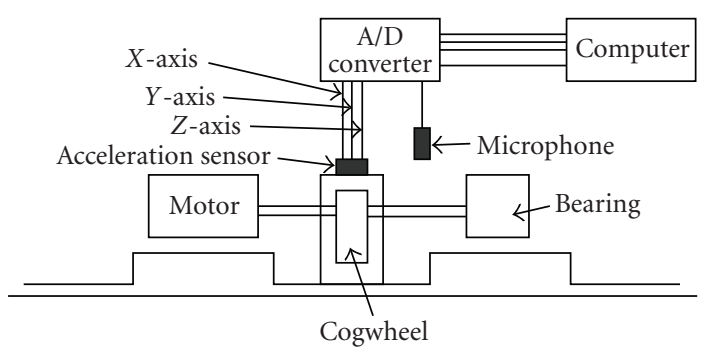

Figure 4: Experimental setup for the measurement of sound and vibration.

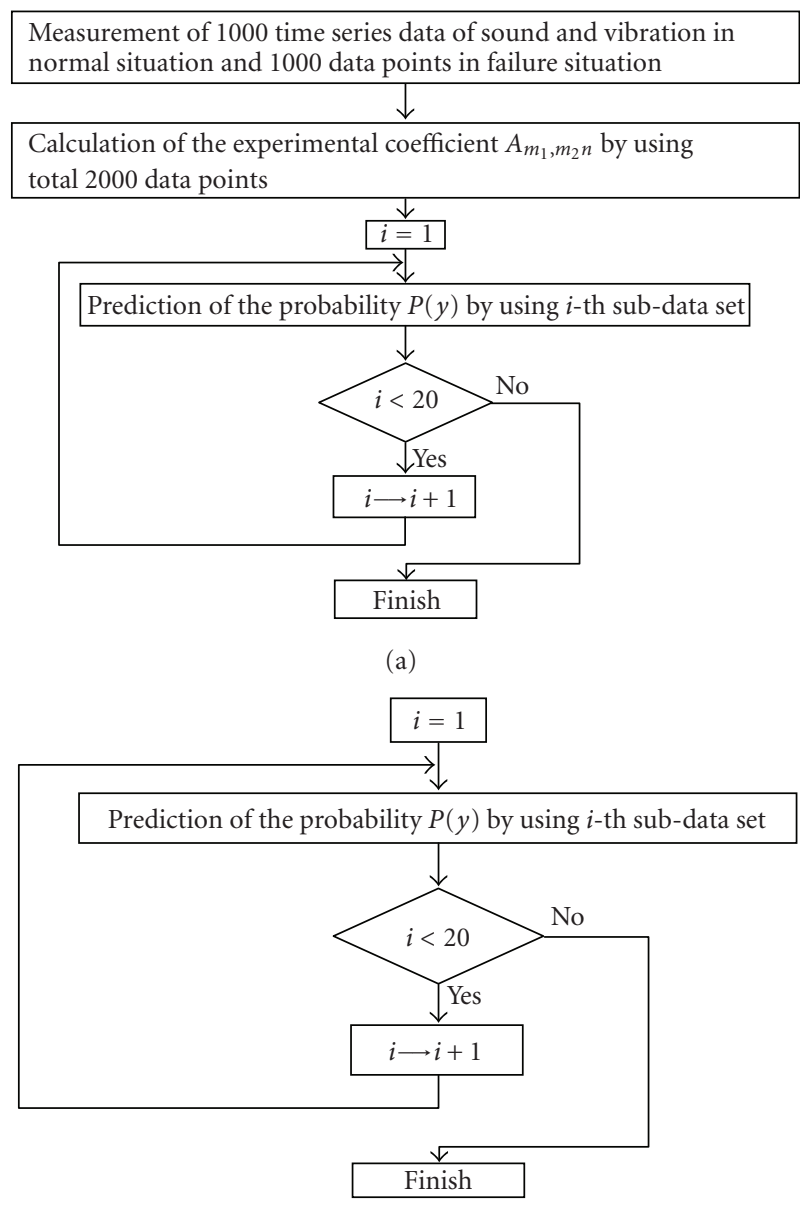

(b)

FIGURE 5: Signal processing procedures for Datasets 1 and 2.

\subsection{Prediction of fault probability based on the observation of sound and vibration}

Based on the conditional probability distribution $P\left(y \mid x_{1}, x_{2}\right)$, the probability $P(y)$ expressing the occurrence or nonoccurrence of a fault can be predicted on the basis of the observation data on sound $x_{1}$ and vibration $x_{2}$ as follows:

$$
P(y)=\left\langle P\left(y \mid x_{1}, x_{2}\right)\right\rangle_{x_{1}, x_{2}}
$$

TABLE 1: Probabilities predicted by using sequentially subdatasets of sound and vibration (for Dataset 1).

\begin{tabular}{lcc}
\hline Data points & $P(y=0)$ & $P(y=1)$ \\
\hline $1-500$ & 0.5333 & 0.4667 \\
$101-600$ & 0.5745 & 0.4255 \\
$201-700$ & 0.5444 & 0.4556 \\
$301-800$ & 0.5169 & 0.4831 \\
$401-900$ & 0.5176 & 0.4824 \\
$501-1000$ & 0.4956 & 0.5044 \\
$601-1100$ & 0.4784 & 0.5216 \\
$701-1200$ & 0.4599 & 0.5401 \\
$801-1300$ & 0.4480 & 0.5520 \\
$901-1400$ & 0.4359 & 0.5641 \\
$1001-1500$ & 0.4266 & 0.5734 \\
$1101-1600$ & 0.4231 & 0.5769 \\
$1201-1700$ & 0.4168 & 0.5832 \\
$1301-1800$ & 0.4087 & 0.5913 \\
$1401-1900$ & 0.4120 & 0.5880 \\
$1501-2000$ & 0.4078 & 0.5922 \\
\hline
\end{tabular}

TABle 2: Probabilities predicted by using sequentially subdatasets of sound and vibration (for Dataset 2).

\begin{tabular}{lcc}
\hline Data points & $P(y=0)$ & $P(y=1)$ \\
\hline $1-500$ & 0.6008 & 0.3992 \\
$101-600$ & 0.5933 & 0.4067 \\
$201-700$ & 0.5728 & 0.4272 \\
$301-800$ & 0.5470 & 0.4530 \\
$401-900$ & 0.5245 & 0.4755 \\
$501-1000$ & 0.5033 & 0.4967 \\
$601-1100$ & 0.4776 & 0.5224 \\
$701-1200$ & 0.4613 & 0.5387 \\
$801-1300$ & 0.4481 & 0.5519 \\
$901-1400$ & 0.4381 & 0.5619 \\
$1001-1500$ & 0.4299 & 0.5701 \\
$1101-1600$ & 0.4216 & 0.5784 \\
$1201-1700$ & 0.4135 & 0.5865 \\
$1301-1800$ & 0.4071 & 0.5929 \\
$1401-1900$ & 0.4021 & 0.5979 \\
$1501-2000$ & 0.3966 & 0.6034 \\
\hline
\end{tabular}

Thus, by the use of (5), $P(y)$ can be expressed concretely as

$$
\begin{gathered}
P(y)=P_{0}(y) \sum_{n=0}^{\infty} B_{n} \varphi_{n}^{(3)}(y) \\
B_{n}=\left\langle\frac{\sum_{m_{1}=0}^{\infty} \sum_{m_{2}=0}^{\infty} A_{m_{1} m_{2} n} \varphi_{m_{1}}^{(1)}\left(x_{1}\right) \varphi_{m_{2}}^{(2)}\left(x_{2}\right)}{\sum_{m_{1}=0}^{\infty} \sum_{m_{2}=0}^{\infty} A_{m_{1} m_{2} 0} \varphi_{m_{1}}^{(1)}\left(x_{1}\right) \varphi_{m_{2}}^{(2)}\left(x_{2}\right)}\right\rangle_{x_{1}, x_{2}} .
\end{gathered}
$$

\section{EXPERIMENT}

The proposed method is applied to detect the fault of a rotational machine by observing simultaneously the sound and vibration waves emitted from the machine. The scatted diagram between the sound and vibration in two cases before 


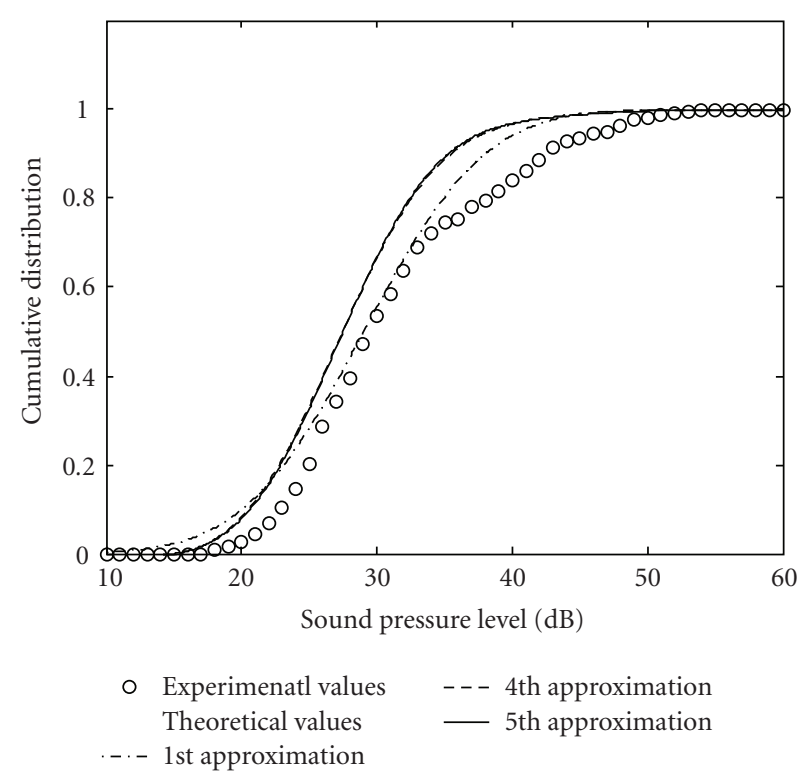

Figure 6: Prediction of the probability distribution for sound pressure level based on the measurement data of vibration in normal situation.

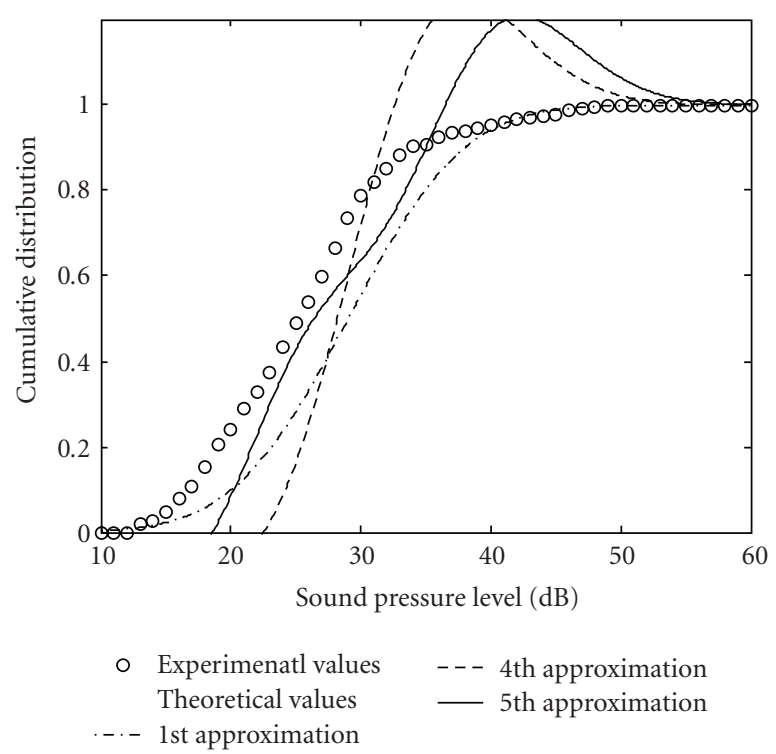

FIgURE 7: Prediction of the probability distribution for sound pressure level based on the measurement data of vibration in failure situation.

and after occurrence of the fault in the machine is shown in Figures 1 and 2, respectively. It is obvious that the correlation relationship between the sound and vibration in the case of failure situation occurring of the fault changes from the correlation characteristic before the occurrence of the fault. Therefore, by detecting the changing information of the correlation characteristic between the sound and vibration, it is possible to predict the fault of the machine in principle. (The sensitivity for the fault detection of the proposed method is discussed in the appendix.)
The signal processing for the observed data by the use of the proposed diagnosis method in Sections 2 and 3 is summarized as follows.

(i) Time series data of sound and vibration waves including two cases of occurrence and nonoccurrence of a fault are simultaneously measured.

(ii) The expansion coefficients $A_{m_{1} m_{2} n}$ in (2) are evaluated by averaging the observed data, concretely, by the use of the relationship

$$
A_{m_{1} m_{2} n}=\frac{1}{S} \sum_{i=1}^{S} \varphi_{m_{1}}^{(1)}\left(x_{1, i}\right) \varphi_{m_{2}}^{(2)}\left(x_{2, i}\right) \varphi_{n}^{(3)}\left(y_{i}\right)
$$

where the expansion coefficients are experimentally estimated. In (17), $S$ is a large number of the observed total data, the subscript $i$ of $x_{1}$ and $x_{2}$ denotes the $i$ th sampled data in time series, and $y_{i}$ is 0 or 1 corresponding to the normal situation without the fault or a failure situation with the fault. Parameters $\mu_{x_{1}}, \sigma_{x_{1}}^{2}, \mu_{x_{2}}, \sigma_{x_{2}}^{2}$ in (7) and $p$ in (12) are estimated by averaging the observed data as

$$
\begin{gathered}
\mu_{x_{1}}=\frac{1}{S} \sum_{i=1}^{S} x_{1, i}, \quad \sigma_{x_{1}}^{2}=\frac{1}{S} \sum_{i=1}^{S}\left(x_{1, i}-\mu_{x_{1}}\right)^{2}, \\
\mu_{x_{2}}=\frac{1}{S} \sum_{i=1}^{S} x_{2, i}, \quad \sigma_{x_{2}}^{2}=\frac{1}{S} \sum_{i=1}^{S}\left(x_{2, i}-\mu_{x_{2}}\right)^{2}, \\
\mu=\frac{1}{S} \sum_{i=1}^{S} y_{i} .
\end{gathered}
$$

(iii) The conditional probability containing the whole information on the regression relationship is concretely evaluated by the use of (5) and the expansion coefficients $A_{m_{1} m_{2} n}$ estimated in (ii).

(iv) In order to predict the probability of the fault occurrence based on the two variables $x_{1}$ and $x_{2}$ (i.e., sound and vibration waves), different kinds of data on $x_{1}$ and $x_{2}$ from those employed in the evaluation of $A_{m_{1} m_{2} n}$ are observed.

(v) By using (16) and using newly observed these data of $x_{1}$ and $x_{2}$ for the prediction of $P(y)$, the expansion coefficients $B_{n}$ can be evaluated as

$$
B_{n}=\frac{1}{T} \sum_{i=1}^{T} \frac{\sum_{m_{1}=0}^{\infty} \sum_{m_{2}=0}^{\infty} A_{m_{1} m_{2} n} \varphi_{m_{1}}^{(1)}\left(x_{1, i}\right) \varphi_{m_{2}}^{(2)}\left(x_{2, i}\right)}{\sum_{m_{1}=0}^{\infty} \sum_{m_{2}=0}^{\infty} A_{m_{1} m_{2} 0} \varphi_{m_{1}}^{(1)}\left(x_{1, i}\right) \varphi_{m_{2}}^{(2)}\left(x_{2, i}\right)},
$$

where $T$ is the number of data observed for the prediction and the subscript $i$ denotes the $i$ th sampled data in time series.

(vi) Thus, by the use of (15), the probability $P(y)$ of the fault occurrence can be predicted.

The aboveprocedure from (iv) to (vi) based on time series data can be carried out easily in online signal processing formby using a personal computer.

The RMS values of the sound pressure level $(\mathrm{dB})$ and the acceleration amplitude $\left(\mathrm{m} / \mathrm{s}^{2}\right)$ emitted from a rotational machine driven by an electric motor are simultaneously measured. The RMS values of the acceleration amplitude are obtained by composing three axis values. More specifically, 
two kinds of time series datasets (i.e., Dataset 1 and Dataset 2 ) of sound and vibration in two different time intervals are measured with a sampling interval of 0.1 second, in two cases of a fault occurrence and nonoccurrence, successively. As an example of faults, the existence of a scratch in a cogwheel is considered as a trial. By adopting the 2000 data points of Dataset 1 which contain both cases of the fault occurrence and nonoccurrence as the learning data, the expansion coefficients are first evaluated by the use of (17) with $m_{1} \leq 3$, $m_{2} \leq 3, n \leq 2$, and $S=2000$. Next, after dividing each dataset into 20 subdatasets which consist of 500 data points with 400 overlapping data points, the probability of the fault occurrence $P(y)$ is predicted by the use of (15) and (19) with $m_{1} \leq 3, m_{2} \leq 3$, and $T=500$ based on the 500-sampled data in each subdataset. The relationship between each dataset and subdatasets is illustrated in Figure 3. The experimental setup and the signal processing procedures for Datasets 1 and 2 are illustrated in Figures 4 and 5.

The predicted results of the probabilities of $y=0$ corresponding to the normal situation without the fault and $y=1$ corresponding to the failure situation with the fault are shown in Table 1 for Dataset 1 and in Table 2 for Dataset 2, respectively. From these results, it is obvious that the fault probability shows clearly large values after the subdatasets in the failure situation are used in order to predict the probability $P(y)$ because the correlation characteristics between sound and vibration are changed after the occurrence of the failure.

\section{CONCLUSION}

In this paper, in order to predict minutely and universally the fault probability based on the mutual relationship between sound and vibration emitted from rotational machine, a method to estimate the correlation information especially in the time domain has been proposed in the form suitable for online signal processing by employing a personal computer. Our proposed method has utilized not only the linear correlation of lower order but also the nonlinear correlation information of higher order between variables. The validity and effectiveness of the proposed method have been confirmed experimentally by applying it to the observation data emitted from a rotational machine.

The proposed method can evaluate numerically the occurrence of a fault as probability. If the fault probability predicted by using (15) and (16) shows larger value, it can be considered that the possibility of the occurrence of fault becomes higher. In this situation, it is necessary to check carefully whether the fault actually occurs or not by stopping the machine. The proposed method has an advantage to evaluate numerically the occurrence of fault in online signal processing form. Furthermore, the fault detection with high reliability can be achieved because the proposed method utilizes total information on sound and vibration.

The proposed approach is obviously quite different from the ordinary approach, and it is still at the early stage of study. Thus, there are a number of problems to be addressed in the future, building on the resultsof the basic study in the paper. Some of the problems are given in the following.
(1) In order to estimate more accurately the occurrence of failures, it is essential to more accurately estimate the conditional probability distribution $P\left(y \mid x_{1}, x_{2}\right)$ given by the expansion expression in (5). From the theoretical viewpoint, the conditional probability distribution can be expressed with higher precision, by employing many expansion coefficients $A_{m_{1} m_{2} n}$ of higher order. From the practical viewpoint, however, the reliability of the conditional probability distribution in the proposed expression tends to be lacking especially for the higher-order correlation information because only a finite numberof data can be observed in practice. It is thus a problem as how the optimal number of terms in the above expansion expression should be determined according to the statistical property of the phenomenon and the available number of data.

(2) The proposed method should be applied to actual diagnoses for other kinds of failures of machines, and the proposed theory should be extended to more realistic situation with many kinds of faults in the machine.

(3) The proposed theory should be further improved to fit the actual situations in the presence of external and internal noises.

\section{APPENDIX}

\section{SENSITIVITY FOR THE FAULT DETECTION}

From (1), the joint probability density functions of $x_{1}$ and $x_{2}$ is obtained as follows:

$$
\begin{aligned}
P\left(x_{1}, x_{2}\right) & =\sum_{y} P\left(x_{1}, x_{2}, y\right) \\
& =P_{0}\left(x_{1}\right) P_{0}\left(x_{2}\right) \sum_{m_{1}=0}^{\infty} \sum_{m_{2}=0}^{\infty} A_{m_{1} m_{2} 0} \varphi_{m_{1}}^{(1)}\left(x_{1}\right) \varphi_{m_{2}}^{(2)}\left(x_{2}\right) .
\end{aligned}
$$

The expansion coefficients $A_{m_{1} m_{2} n}$ are defined by (2). Then, the conditional probability density function can be given by

$$
\begin{aligned}
P\left(x_{1} \mid x_{2}\right) & =\frac{P\left(x_{1}, x_{2}\right)}{P\left(x_{2}\right)} \\
& =\frac{P_{0}\left(x_{1}\right) \sum_{m_{1}=0}^{\infty} \sum_{m_{2}=0}^{\infty} A_{m_{1} m_{2} 0} \varphi_{m_{1}}^{(1)}\left(x_{1}\right) \varphi_{m_{2}}^{(2)}\left(x_{2}\right)}{\sum_{m_{2}=0}^{\infty} A_{0 m_{2} 0} \varphi_{m_{2}}^{(2)}\left(x_{2}\right)} .
\end{aligned}
$$

The probability density function of $x_{1}$ can be predicted on the basis of the observed data of $x_{2}$ as follows:

$$
\begin{aligned}
P\left(x_{1}\right) & =\left\langle P\left(x_{1} \mid x_{2}\right)\right\rangle_{x_{2}} \\
& =P_{0}\left(x_{1}\right) \sum_{m_{1}=0}^{\infty}\left\langle\frac{\sum_{m_{2}=0}^{\infty} A_{m_{1} m_{2} 0} \varphi_{m_{2}}^{(2)}\left(x_{2}\right)}{\sum_{m_{2}=0}^{\infty} A_{0 m_{2} 0} \varphi_{m_{2}}^{(2)}\left(x_{2}\right)}\right\rangle_{x_{2}} \varphi_{m_{1}}^{(1)}\left(x_{1}\right) .
\end{aligned}
$$

The probability density function in orthogonal expansion series has theoretically convergent property by considering expansion terms with higher orders. Therefore, after evaluating in advance the expansion coefficients $A_{m_{1} m_{2} 0}$ by the 
use of the measured data on sound and vibration in normal situation without fault, by using (A.3), the prediction of $P\left(x_{1}\right)$ can be obtained form themeasured data of $x_{2}$ in the normal situation as shown in Figure 6 . On the other hand, by using the measured data $x_{2}$ in a failure situation with the fault, the predicted probability density function $P\left(x_{1}\right)$ shows the tendency to diverge, as shown in Figure 7. Therefore, by introducing an indicator expressing the difference between two probability density functions shown in Figures 6 and 7, it is possible to evaluate quantitatively the occurrence of the fault. It remains to continue as one of future works.

\section{REFERENCES}

[1] S.-N. Gu, J.-L. Zhang, J.-S. Jiang, and C.-A. He, "A vibration diagnosis approach to structural fault," Journal of Vibration, Acoustics, Stress, and Reliability in Design, vol. 111, no. 1, pp. 88-93, 1989.

[2] R. D. Adams, P. Cawley, C. J. Pye, and B. J. Stone, "A vibration technique for non-destructively assessing the integrity of structures," Journal of Mechanical Engineering Science, vol. 20, no. 2, pp. 93-100, 1978.

[3] P. Gundundson, "Eigen frequency changes of structures due to cracks noteches or toehr geometrical changes," Journal of the Mechanics and Physics of Solids, vol. 30, no. 5, pp. 339-353, 1982.

[4] H. Kanai, M. Abe, and K. Kido, "Detection and discrimination of flaws in ball bearings by vibration analysis," Journal of the Acoustical Society of Japan E, vol. 7, no. 2, pp. 121-131, 1986.

[5] G. Zurita-Villarroel and A. Ågren, "A new approach to diagnostics of the combustion Process in Diesel Engines using vibration measurements-prat I: reconstruction of cylinder pressure form vibration measurements," International Journal of Acoustics and Vibration, vol. 8, no. 2, pp. 68-76, 2003.

[6] L. Gelman, P. Jenkin, M. Sanderson, C. Thompson, and M. J. Crocker, "A new generalised approach for feature representation in vibroacoustical fault diagnosis," International Journal of Acoustics and Vibration, vol. 9, no. 1, pp. 13-16, 2004.

[7] F. Bonnardot, R. B. Randall, and J. Antoni, "Unsupervised angular resampling and noise cancellation for planetary bearing fault diagnosis," International Journal of Acoustics and Vibration, vol. 9, no. 2, pp. 51-60, 2004.

[8] J. K. Sinha and A. R. Rao, "Vibration diagnosis of failure of mechanical coupling between motor and pump rotors," International Journal of Acoustics and Vibration, vol. 10, no. 2, pp. 89-92, 2005.

[9] L. Gelman, P. Jenkin, I. Petrunin, and M. J. Crocker, "Vibroacoustical damping diagnostics: complex frequency response function versus its magnitude," International Journal of Acoustics and Vibration, vol. 11, no. 3, pp. 120-124, 2006.

[10] M. Ohta and T. Koizumi, "General statistical treatment of the response of a non-lineae rectifying device to a stationary random input," IEEE Transactions on Information Theory, vol. 14, no. 4, pp. 595-598, 1968.

[11] G. R. Cooper and C. D. McGillem, Probabilistic Methods of Signal and System Analysis, Oxford University Press, Oxford, UK, 1999.

[12] M. Ohta, A. Ikuta, and N. Takaki, "An evaluation method for an arbitrary probability distribution with digital level observation in sound and vibration systems," Acustica, vol. 67, pp. 73-85, 1988.

[13] H. Cramer, Mathematical Methods of Statistics, Princeton University Press, Princeton, NJ, USA, 1951. 

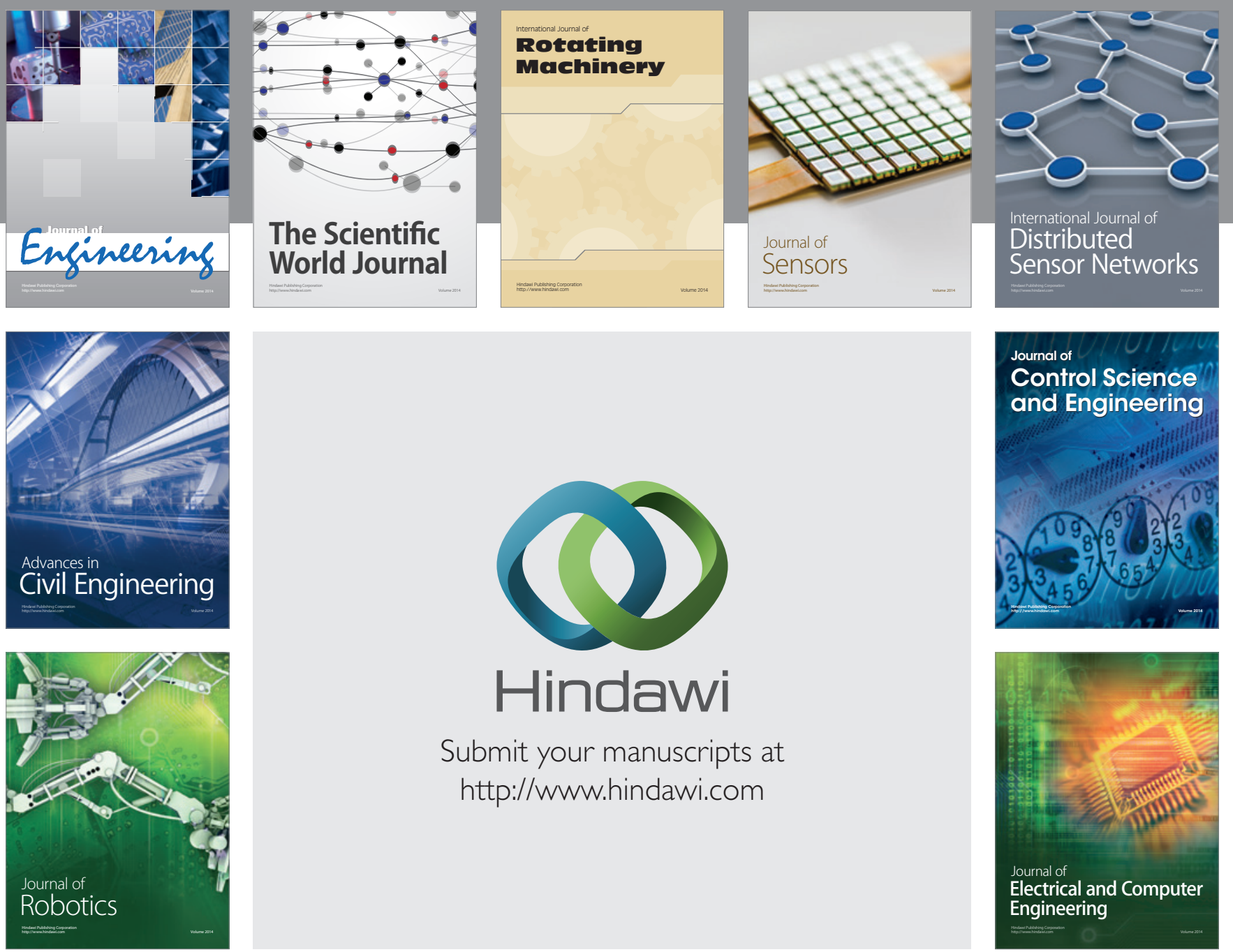

Submit your manuscripts at

http://www.hindawi.com
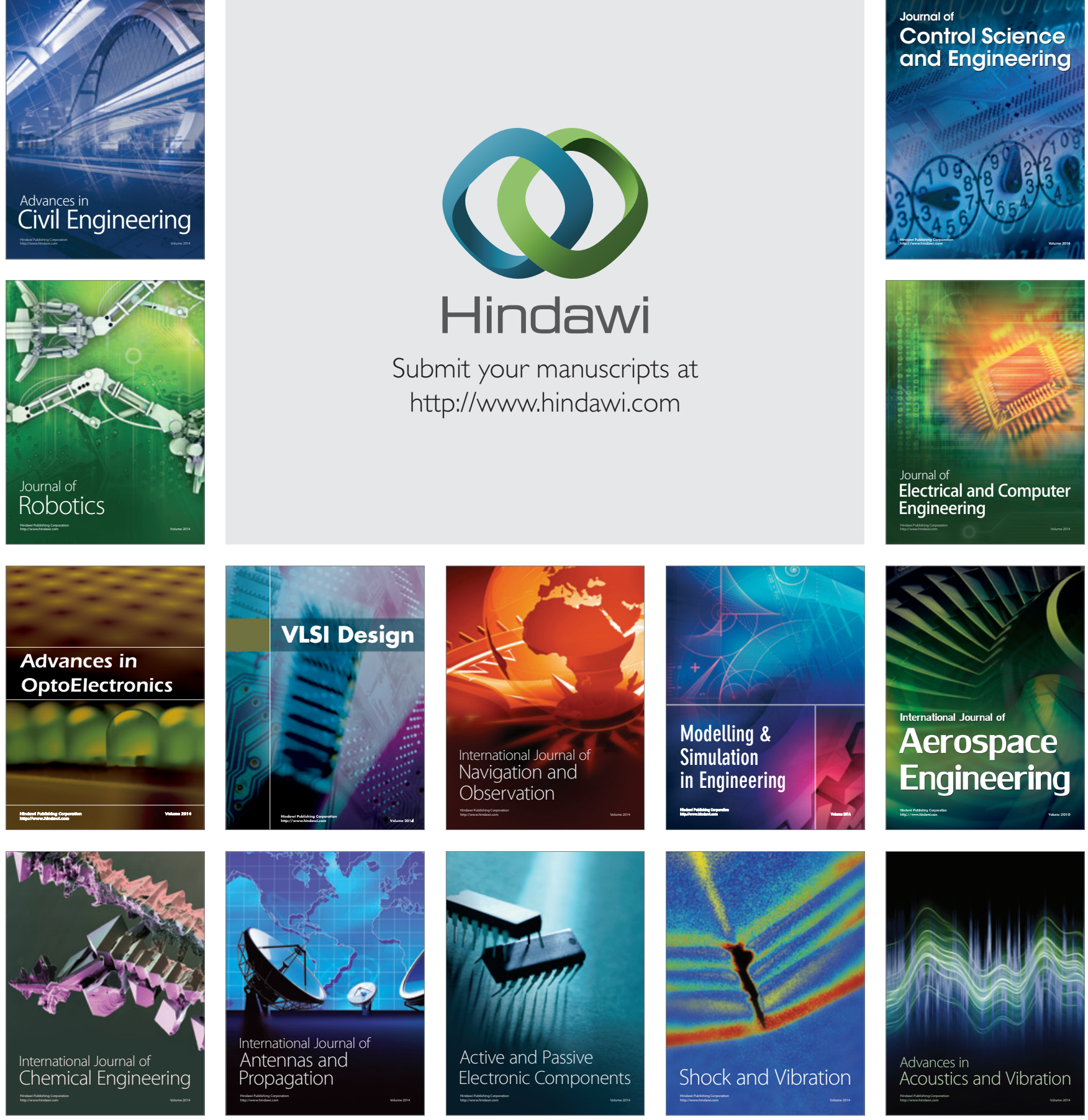